\title{
BMJ Open Differences in problem alcohol drinking by military service type among male professional military personnel in South Korea using Military Health Survey data
}

\author{
Jeongok Park, ${ }^{1}$ Eunyoung Jung (D) , ${ }^{1,2}$ Eunkyoung Bae ${ }^{1}$
}

To cite: Park J, Jung E, Bae E. Differences in problem alcohol drinking by military service type among male professional military personnel in South Korea using Military Health Survey data. BMJ Open 2021;11:e045279. doi:10.1136/ bmjopen-2020-045279

- Prepublication history for this paper is available online. To view these files, please visit the journal online (http://dx.doi. org/10.1136/bmjopen-2020045279).

Received 07 October 2020 Accepted 21 August 2021

Check for updates

(C) Author(s) (or their employer(s)) 2021. Re-use permitted under CC BY-NC. No commercial re-use. See rights and permissions. Published by BMJ.

${ }^{1}$ Mo-Im Kim Nursing Research Institute, College of Nursing, Yonsei University, Seoul, Republic of Korea

${ }^{2}$ Korea Armed Forces Nursing Academy, Daejeon, Republic of Korea

Correspondence to Dr Eunyoung Jung; eunyoung1708@gmail.com

\section{ABSTRACT}

Objectives Alcohol drinking prevalence in Korea is higher than in other countries and is associated with various social and health problems. Korean culture tends to be tolerant of alcohol drinking and to regard it as an important medium for maintaining good interpersonal relationships in one's social life. Although alcohol drinking is a means of relieving stress, especially among soldiers, who engage in more binge drinking than civilians, there is lack of research focused on problem drinking among soldiers. Therefore, the purpose of this study was to explore the extent of problem drinking across all military services and to identify factors associated with problem drinking by military service type.

Design Secondary data analysis.

Setting Dataset of the Military Health Survey in 2015. Participants Altogether, 2252 male professional military personnel were included in this study.

Main outcome measure Problem drinking in this study was defined as at-risk drinking and alcohol abuse or dependence with an Alcohol Use Disorders IdentificationKorean (AUDIT-K) score of 10 points or higher.

Results The average AUDIT-K score was $7.38 \pm 4.10$ points. The prevalence of problem drinking was $16.4 \%$ for the Army, 34.5\% for the Navy and $32.1 \%$ for the Air Force. Factors associated with problem drinking for each military service type were sleep satisfaction (OR 2.33, $95 \% \mathrm{Cl} 1.284$ to 4.236 ) and family support (OR $0.66,95 \%$ $\mathrm{Cl} 0.487$ to 0.904 ) in the Army, smoking status (OR 1.85, $95 \% \mathrm{Cl} 1.130$ to 3.039) and sleep satisfaction (OR 2.29, $95 \% \mathrm{Cl} 1.142$ to 4.574$)$ in the Navy and marital status (OR $0.60,95 \% \mathrm{Cl} 0.382$ to 0.951 ), smoking (past smokers and non-smokers OR 2.81, 95\% Cl 1.593 to 4.973 , current smokers and non-smokers OR $1.68,95 \% \mathrm{Cl} 1.114$ to 2.544), subjective oral health (OR $1.83,95 \% \mathrm{Cl} 1.011$ to 3.297 ) and family support (OR $0.63,95 \% \mathrm{Cl} 0.45$ to 0.88 ) in the Air Force.

Conclusion When implementing health projects to address drinking problems, it is necessary to ensure that service-type-specific factors are considered for integrated management.

\section{BACKGROUND}

Alcohol drinking prevalence in Korea is higher than in other countries and is associated with various social and health problems.

\section{Strengths and limitations of this study}

- This study examined factors affecting problem alcohol drinking by military service type among male professional military personnel in South Korea.

- Affecting factors were identified from various aspects including individual characteristics, military characteristics, health behaviours and psychological health.

- This study was conducted through a self-report survey, which may have resulted in reporting bias, especially for questions on alcohol drinking.

In 2015, Korea's alcohol consumption per person aged 15 or older stood at $9.1 \mathrm{~L} /$ year, higher than the average of $9.0 \mathrm{~L}$ in Organisation for Economic Co-operation and Development (OECD) countries. ${ }^{1}$ In addition, monthly binge drinking prevalence for Korean men was $52.7 \%$ in $2017 .{ }^{2}$ On average, $3.7 \%$ of adults in OECD countries were heavily dependent on alcohol in 2016; this figure stood at $5.5 \%$ for Korea in $2015 .^{3}$

In Korea, the social atmosphere is tolerant of alcohol drinking behaviour. Alcohol drinking is recognised as an important medium for maintaining good interpersonal relationships in social life. ${ }^{5}$ Most people enjoy drinking with others, and mixing different liquors, such as in bomb shots, is common. ${ }^{6}$ Such a culture in which drinking is commonplace makes it difficult for people to express their opinions comfortably in situations where they do not want to drink or when they must quit drinking. ${ }^{5}$ Military personnel tend to rely on alcohol drinking as a means of relieving stress; those who prepare for combat tend to have a higher level of stress than the general population. ${ }^{78}$ Military personnel are known to engage in more binge drinking than civilians. $^{7910}$ Therefore, there is an increasing need to create a safe drinking culture. 
Problem alcohol drinking is defined as drinking behaviour that causes issues in the drinker's life or in the life of someone they know; this refers not only to physical problems such as liver disease, but also relationships with family, friends and colleagues. ${ }^{11}$ Problem alcohol drinking among military personnel causes difficulties not only with health but also with job performance. ${ }^{10}$ Previous studies have linked problem alcohol drinking among military personnel to sociodemographic characteristics such as gender, age and marital status; military-related characteristics such as military service type and healthrelated characteristics such as smoking, injury and stress levels. ${ }^{7}{ }^{12-15}$ Recent research has been conducted on military personnel's drinking in the context of individual military service groups. ${ }^{8} 1215-17$ Personnel from different military services show different drinking behaviours in terms of frequency and amount, owing to differences in eligibility criteria for recruitment, occupational roles and drinking cultures. ${ }^{121718}$

In Korea, most studies on alcohol drinking have examined it as part of health behaviour, and there is a lack of research focused on problem alcohol drinking, especially among military personnel. ${ }^{19-21}$ In accordance with the Law for the Promotion of Nation's Health established in 1995, Korean nursing officers have conducted health promotion projects to preserve military personnel's health and military combat capabilities since 2000. ${ }^{22}{ }^{23}$ Although the military has consistently implemented drinking-related projects, military personnel's drinking behaviours have shown a similar pattern without major change. ${ }^{72124}$ This seems to be because the military has carried out general campaigns without considering drinking behaviour and its associated factors by military service type in Korea. To foster a safe drinking culture within the military and to increase the effectiveness of drinking-related health programmes, it is necessary to examine the current status of drinking among military personnel and its associated factors. Therefore, the purpose of this study was to explore the extent of problem alcohol drinking across all military services and to identify factors associated with problem alcohol drinking by military service type in South Korea.

\section{METHODS}

\section{Study design}

This study was a secondary data analysis using the dataset of the 2015 Military Health Survey (MHS) conducted by the Republic of Korea (ROK) School of Military Medicine.

\section{Data source}

The MHS was conducted for only 2 years, from 2014 to 2015 , to understand the health behaviour and medical utilisation of military personnel. The survey consisted of four domains: health behaviour, injury/safety, mental health and medical utilisation. It was designed to fit the situation of the military based on the Korean National Health and Nutrition Survey. In consideration of the fact that the survey measurement tools for the major variables in 2014 and 2015 were different, this study only used data from 2015. The MHS used stratified random sampling considering professional military personnel's sex, rank, service type and work area to represent the entire population of ROK military personnel including professional and enlisted soldiers in 2015. In Korea, professional military personnel are those who choose military service as a career, and in this study, the term refers to officers or non-commissioned officers. The survey questionnaire was distributed to 9520 personnel, and 6395 surveys were returned. With the exclusion of 113 incomplete responses, 6282 surveys were compiled into a dataset that included information on health behaviours such as smoking, drinking and physical activity, injury and safety awareness, quality of life and mental health, social support, medical health service use and personal characteristics.

Enlisted soldiers are not allowed to drink during their military service; hence, they did not respond to alcoholrelated items. Therefore, this study included only professional military personnel. Of the 2785 professional military personnel who participated in the survey, 201 female military personnel and 262 male military personnel who did not answer questions on drinking were excluded. On the scale used in this study, men and women face a different cut-off score ${ }^{25}$; moreover, women were excluded from the study due to the substantial difference in the number of people in each group. In addition, 70 male military personnel of the rank of warrant officer, colonel and general were also excluded because of the different distributions by military service type. Finally, 2252 male professional military personnel were included in this study (figure 1).

\section{Measurements \\ Dependent variable}

The dependent variable was problem alcohol drinking, which refers to drinking behaviour that causes physical, psychological and social problems. ${ }^{11}$ Problem alcohol drinking was defined using the Alcohol Use Disorders Identification Test (AUDIT). AUDIT is a reliable and valid screening tool for problem alcohol drinking. It was

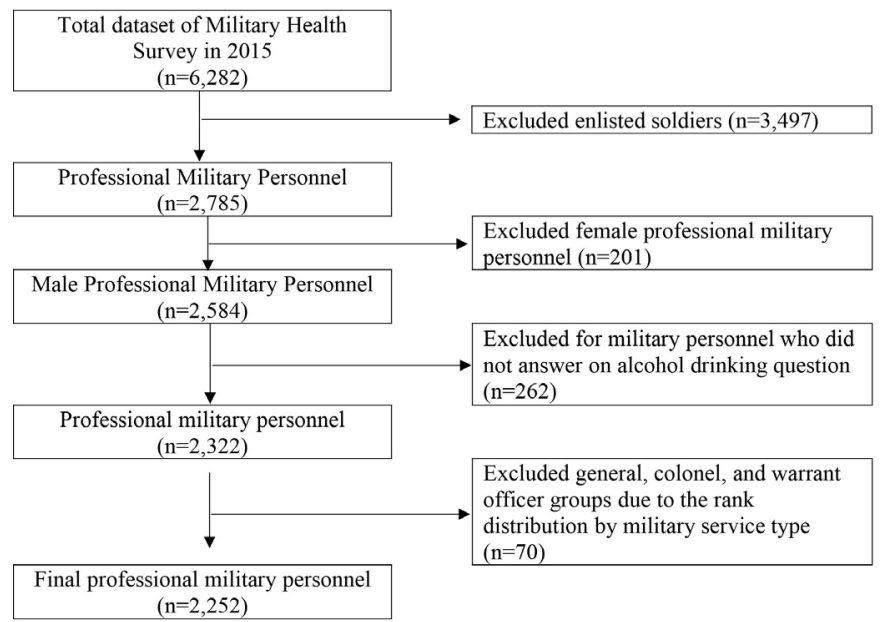

Figure 1 Flow chart of participant in this study. 
developed by the WHO in 1989 and revised in 2001. It consists of 10 items on hazardous alcohol consumption (frequency of drinking, typical quantity and frequency of heavy drinking), dependence symptoms (impaired control over drinking, increased salience of drinking and morning drinking) and harmful alcohol consumption (guilt after drinking, blackouts, alcohol-related injuries and others concerned about drinking).$^{26}$ The items are rated on a five-point Likert scale ( $0-4$ points) for a minimum score of 0 and a maximum score of 40 .

An AUDIT-Korean version (AUDIT-K) score of 10 or more is interpreted as alcohol use disorder for men. ${ }^{25}$ The Ministry of Health and Welfare and Korea Health Promotion Institute has classified drinking types into three groups: the normal drinking group (0-9 points on AUDIT-K), the at-risk drinking group (10-19 points) and the alcohol abuse or dependence group ( $\geq 20$ points).

The operational definition of problem alcohol drinking in this study was at-risk drinking and alcohol abuse or dependence with an AUDIT-K score of 10 points or higher. In this study, we divided participants into two groups: a normal alcohol drinking group (0-9 points) and a problem alcohol drinking group (10-40 points). With respect to scale reliability, Cronbach's $\alpha$ for AUDIT-K was 0.755 in this study.

\section{Independent variables}

The independent variables were individual characteristics, military characteristics, health behaviours and psychological health.

Individual characteristics included age, education level (middle and high school, college and university, graduate school), marital status (married and living with family, married and living apart from family and singledivorced or unmarried) and body mass index (BMI, in accordance with criteria of the Asia-Pacific Perspective). ${ }^{27}$

Military characteristics included military service type (Army, Navy or Air Force), rank (officer-lieutenant colonel or major, captain and first or second lieutenant; non-commissioned officer-sergeant major or master sergeant and sergeant first class or staff sergeant), branch (combat, technical service, administrative support, etc), work area (metropolitan cities, cities, rural areas and isolated military areas) and work type (day, evening, night duty and shift work).

Health behaviours included smoking status (nonsmoker, past smoker and current smoker), aerobic exercise (yes=more than 2 hours and $30 \mathrm{~min}$ of moderate physical activity or 1 hour and $15 \mathrm{~min}$ of high physical activity or mixed moderate and high physical activity ( $1 \mathrm{~min}$ of high physical activity is calculated as $2 \mathrm{~min}$ of moderate physical activity) a week in accordance with the sixth Korea National Health and Nutrition Examination Survey (KNHANES)), ${ }^{28}$ subjective health status and oral health status, sleep hours (in accordance with National Sleep Foundation), and sleep satisfaction. Subjective health status, subjective oral health status and sleep satisfaction were rated on a five-point Likert scale (very poor, poor, moderate, good and very good). The five categories were collapsed into poor (very poor and poor), moderate and good (good and very good).

Psychological health involved occupational life stress, personal life stress, depression and social support from superior soldiers, colleagues, family and friends. Occupational life stress and personal life stress were one item each and were reported on a five-point scale (no, not much, moderate, high and very high). Beck's Depression Inventory (BDI) was used to measure depression; it consists of 21 items rated on a four-point Likert scale. The higher the score, the higher the level of depression. A BDI score greater than 21 was considered as having depression. With respect to scale reliability, Cronbach's $\alpha$ for BDI was 0.905 in this study. Social support involved four items rated on a five-point Likert scale; the higher the score, the better the social support.

\section{Patient and public involvement}

There was no patient or public involvement in this study.

\section{Data analysis}

SPSS statistics 25.0 was used for data analysis (IBM, South Korea). Descriptive analyses were used to assess the study variables, including mean, standard deviation and frequency. Differences in independent variables by military service type were evaluated using a $\chi^{2}$ test and analysis of variance. Multiple logistic regressions were performed, including only significant variables in the univariate analysis, to analyse the associated factors of problem alcohol drinking by military service type. Variance inflation factor (VIF) and tolerance were also checked to identify the correlation among independent variables included in logistic regression.

\section{RESULTS}

\section{General characteristics of participants}

General participant characteristics by military service type are presented in table 1 . Of the total 2252 participants, 979 were from the Army (43.5\%), 550 from the Navy (24.4\%) and 723 from the Air Force $(32.1 \%)$. Regarding individual characteristics, there were significant differences in personnel age $(\mathrm{F}=7.06, \mathrm{p}=0.001)$, education level $\left(x^{2}=137.00, \mathrm{p}<0.001\right)$, and marital status $\left(x^{2}=24.54\right.$, $\mathrm{p}<0.001)$ by military service type. The average BMI was $24.45 \mathrm{~kg} / \mathrm{m}^{2}\left(\mathrm{SD}=2.93\right.$, range $\left.=17-38 \mathrm{~kg} / \mathrm{m}^{2}\right)$.

Participants included 1509 non-commissioned officers $(67.0 \%)$ and 743 officers (33.0\%). The Army $(83.5 \%)$ and Navy (82.4\%) accounted for the highest proportion of personnel in combat, while the Air Force had the highest proportion in technical service $(57.2 \%)$. Army, Navy and Air Force personnel showed significant differences with respect to rank $\left(x^{2}=222.05, \mathrm{p}<0.001\right)$, branch $\left(x^{2}=784.72\right.$, $\mathrm{p}<0.001)$, and work area $\left(x^{2}=769.55, \mathrm{p}<0.001\right)$.

Regarding health behaviours, the most common smoking status was non-smoker $(43.0 \%)$ or current smoker $(44.4 \%)$. A total of $6.3 \%$ participants reported poor subjective health, while $20.1 \%$ reported poor subjective 


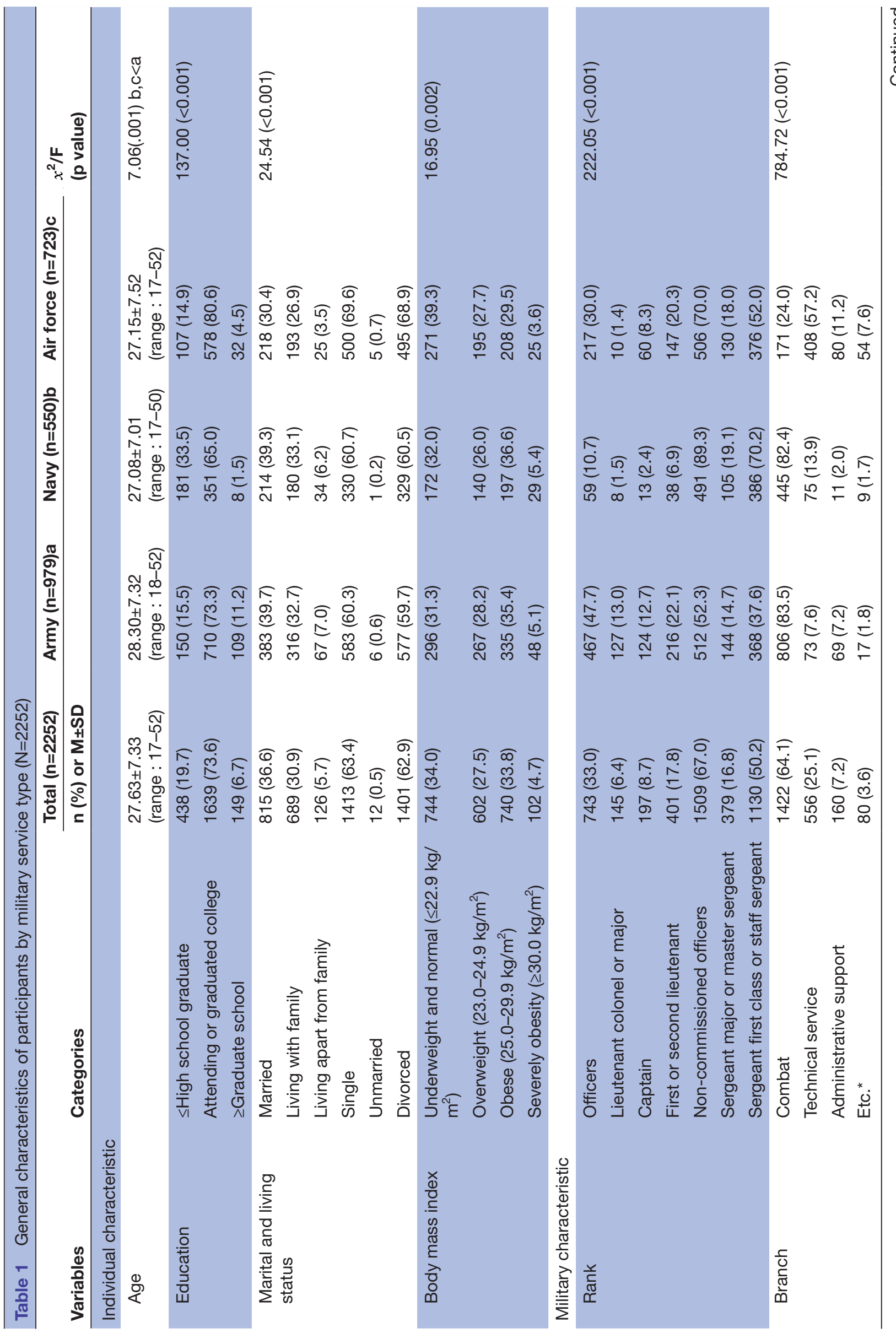




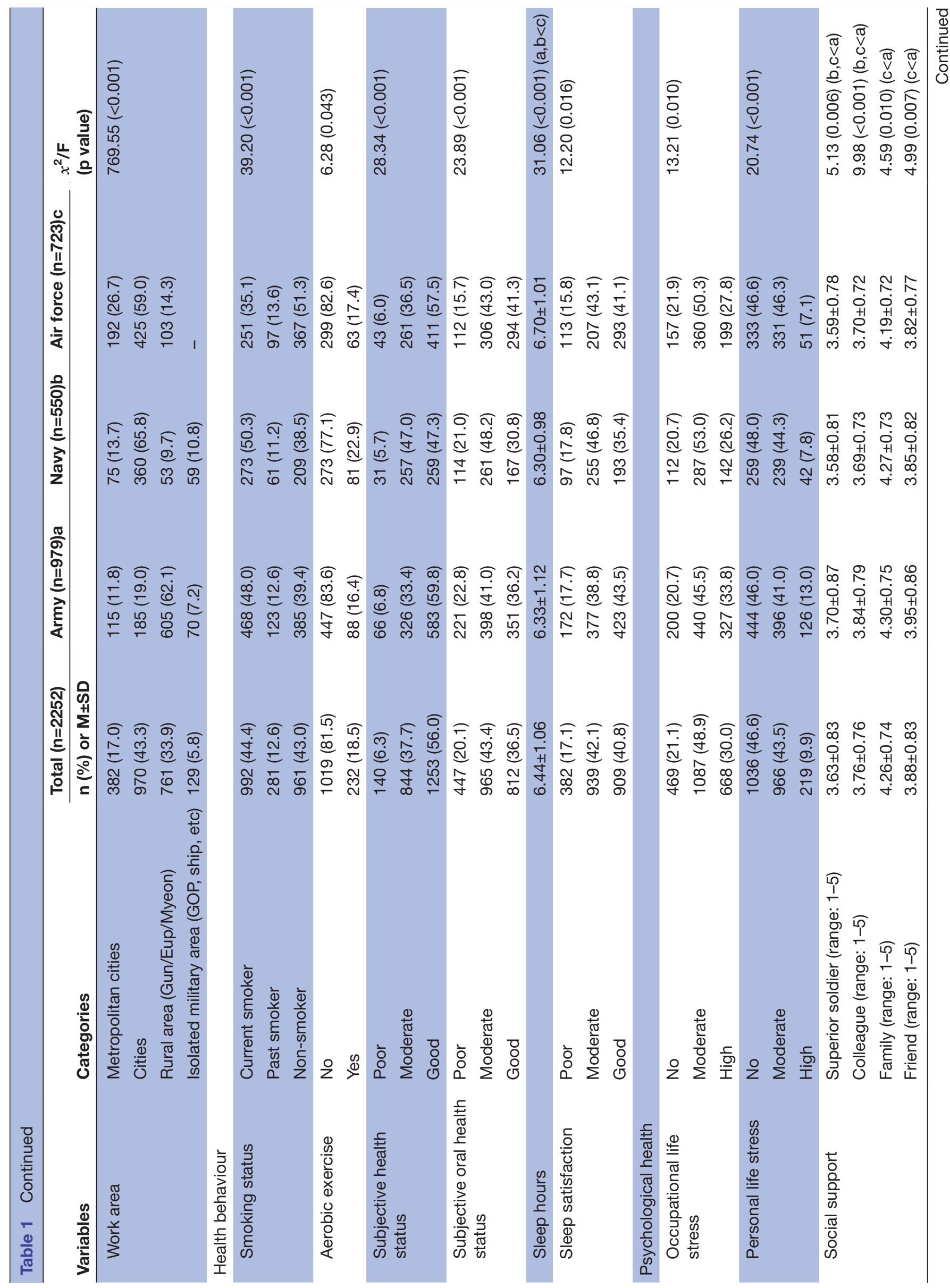




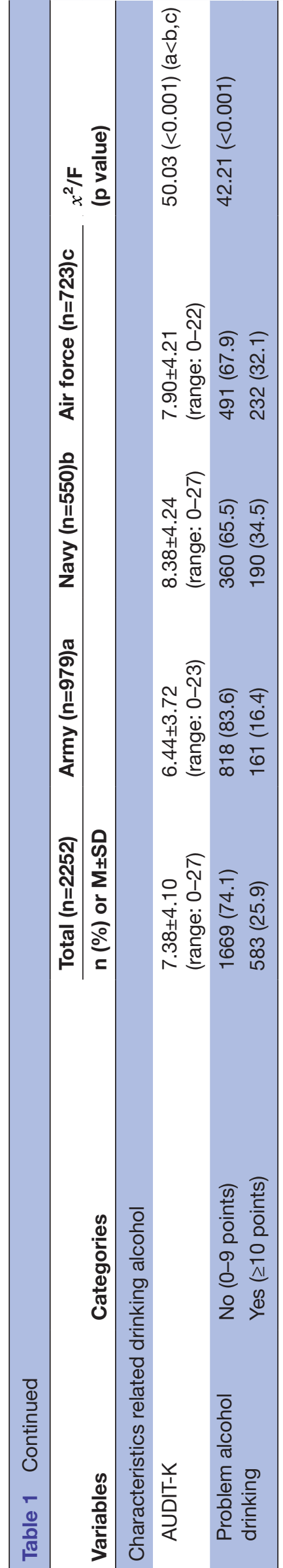

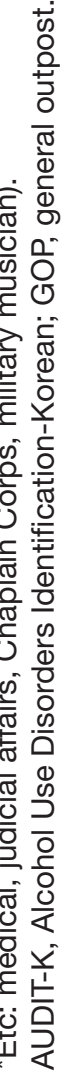

oral health. Army, Navy and Air Force personnel showed significant differences in smoking status $\left(x^{2}=39.20\right.$, $\mathrm{p}<0.001)$, aerobic exercise $\left(x^{2}=6.28, \mathrm{p}=0.043\right)$, subjective health status $\left(x^{2}=28.34, \mathrm{p}<0.001\right)$, subjective oral health status $\left(x^{2}=23.89, \mathrm{p}<0.001\right)$, sleep hours $\left(x^{2}=31.06\right.$, $\mathrm{p}<0.001)$ and sleep satisfaction $\left(x^{2}=12.20, \mathrm{p}=0.016\right)$.

Regarding psychological health, Army, Navy and Air Force personnel showed significant differences in occupational life stress $\left(x^{2}=13.21, \mathrm{p}=0.010\right)$, personal life stress $\left(x^{2}=20.74, \mathrm{p}<0.001\right)$, support from superior soldiers $\left(x^{2}=5.13, \mathrm{p}=0.006\right)$, support from colleagues $\left(x^{2}=9.98\right.$, $\mathrm{p}<0.001)$, support from family $\left(x^{2}=4.59, \mathrm{p}=0.010\right)$ and support from friends $\left(x^{2}=4.99, \mathrm{p}=0.007\right)$.

According to military service type, the average AUDIT-K scores for Army, Navy and Air Force personnel were 6.44 ( $\mathrm{SD}=3.72$ points), $8.38(\mathrm{SD}=4.24)$ and $7.90 \quad(\mathrm{SD}=4.21)$, respectively. The average AUDIT-K score in the Army was significantly lower than those in the Navy and Air Force $(\mathrm{F}=50.03, \mathrm{p}<0.001)$. Similarly, the overall prevalence of problem alcohol drinking was $25.9 \%$, while individual figures for the Army, Navy and Air Force were 16.4\%, $34.5 \%$ and $32.1 \%$, respectively $\left(x^{2}=42.21, \mathrm{p}<0.001\right)$.

The distribution of AUDIT-K scores by military service type and rank are shown using box and violin plots in figure 2. The non-commissioned officers' average AUDIT-K score was higher than officers' across all military service types, but there were no significant differences in AUDIT-K scores by rank in each military service type. The subgroup with the highest AUDIT-K score overall was lieutenant colonel or major of the Navy (mean=9.75, SD=2.61). The subgroup with the lowest AUDIT-K score overall was first or second lieutenant of the Army (mean=5.86, $\mathrm{SD}=3.14$ ). An AUDIT-K score $\geq 20$, which refers to alcohol abuse or dependence, was found in the sergeant first class or staff sergeant group of the Army, sergeant major or master sergeant and sergeant first class or staff sergeant group of the Navy, and first or second lieutenant and sergeant major or master sergeant and sergeant first class or staff sergeant group of the Air Force.

\section{Factors associated with problem alcohol drinking by military service type}

The results of factors associated with problem alcohol drinking by military service type are presented in table 2 . For the multiple logistic regression analysis, we only considered variables showing statistical significance in the univariate analysis. In addition, the VIF was from 1.021 to 2.310 , and the tolerance was from 0.408 to 0.980 , which means that collinearity among independent variables can be ignored.

First, in the Army, after controlling for other factors, people with poor sleep satisfaction had 2.33 times higher problem alcohol drinking than those with good sleep satisfaction (95\% CI 1.284 to 4.236). The higher the support from family, the lower the problem alcohol drinking (OR $0.66,95 \%$ CI 0.487 to 0.904 ). 

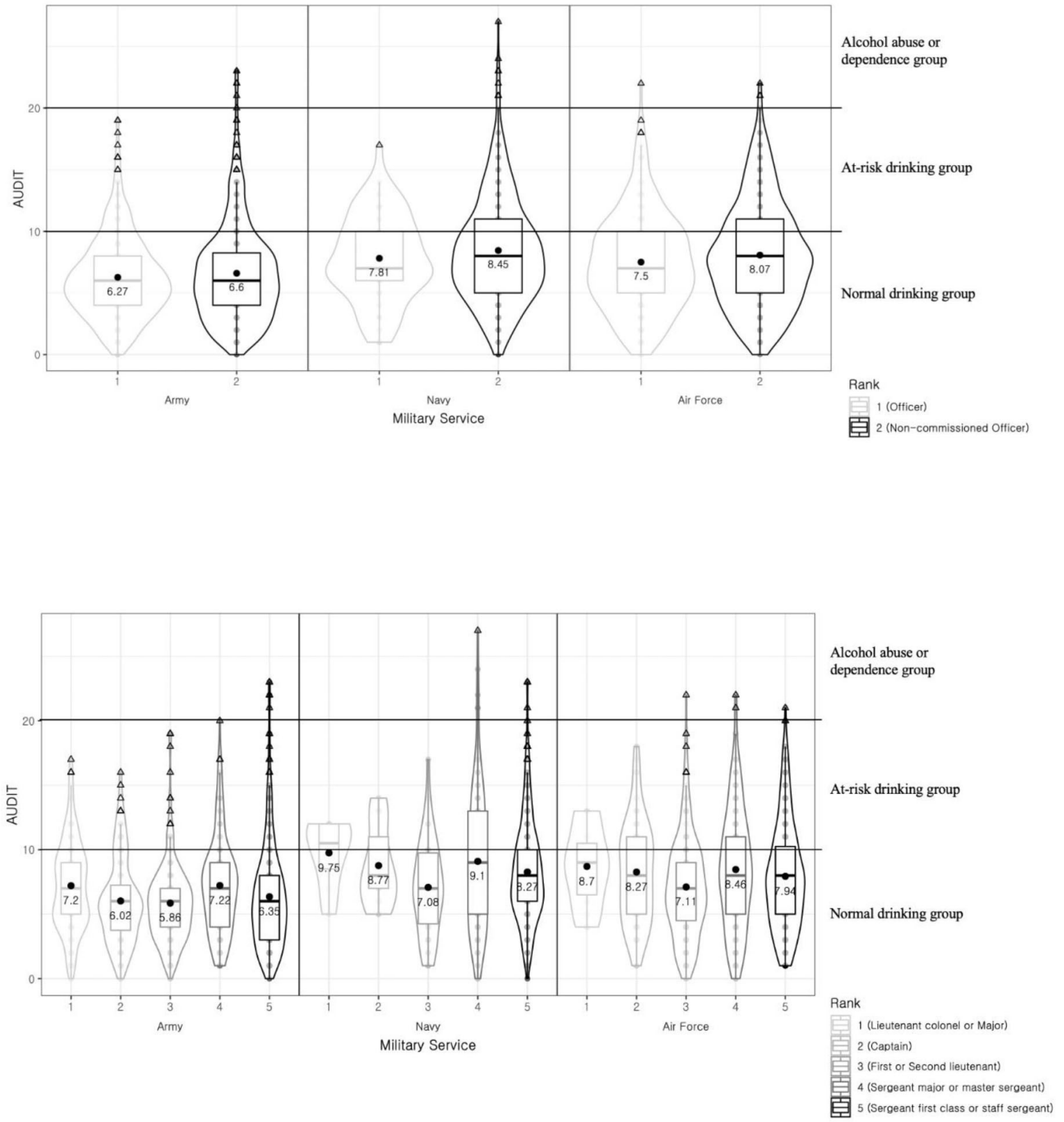

Figure 2 Box and violin plot of AUDIT score by military service type and rank group.

Second, in the Navy, current smokers had 1.85 times higher problem alcohol drinking than non-smokers $(95 \%$ CI 1.130 to 3.039). The poor sleep satisfaction group was more likely to engage in problem alcohol drinking compared with the good sleep satisfaction group (OR $2.29,95 \%$ CI 1.142 to 4.574 ).

Third, in the Air Force, those who were single were less likely to engage in problem alcohol drinking compared with those who were married and living with their family (OR $0.60,95 \%$ CI 0.382 to 0.951 ). Past smokers and current smokers had 2.81 (95\% CI 1.593 to 4.973$)$ and 1.68 (95\% CI 1.114 to 2.544) times higher problem alcohol drinking than non-smokers. People with poor subjective oral health were more likely to engage in problem alcohol drinking than those with good subjective oral health (OR $1.83,95 \%$ CI 1.011 to 3.297$)$. The higher the support from family, the lower the problem alcohol drinking (OR $0.63,95 \%$ CI 0.45 to 0.88 ). However, no military characteristics were identified as factors associated with problem alcohol drinking in any of the service types.

\section{DISCUSSION}

This study was conducted to explore drinking status and examine factors affecting problem alcohol drinking by military service type among male professional military personnel in South Korea. The participants' AUDIT-K scores were similar to those of Korean adult males. The overall AUDIT-K score in this study was $7.38(\mathrm{SD}=4.10)$. The non-alcoholic group's AUDIT-K score was 7.05 $(\mathrm{SD}=4.34),{ }^{29}$ and the AUDIT-K score for men using the KNHANES in 2012 was $8.60(\mathrm{SD}=7.19) .{ }^{30}$ In this study, 


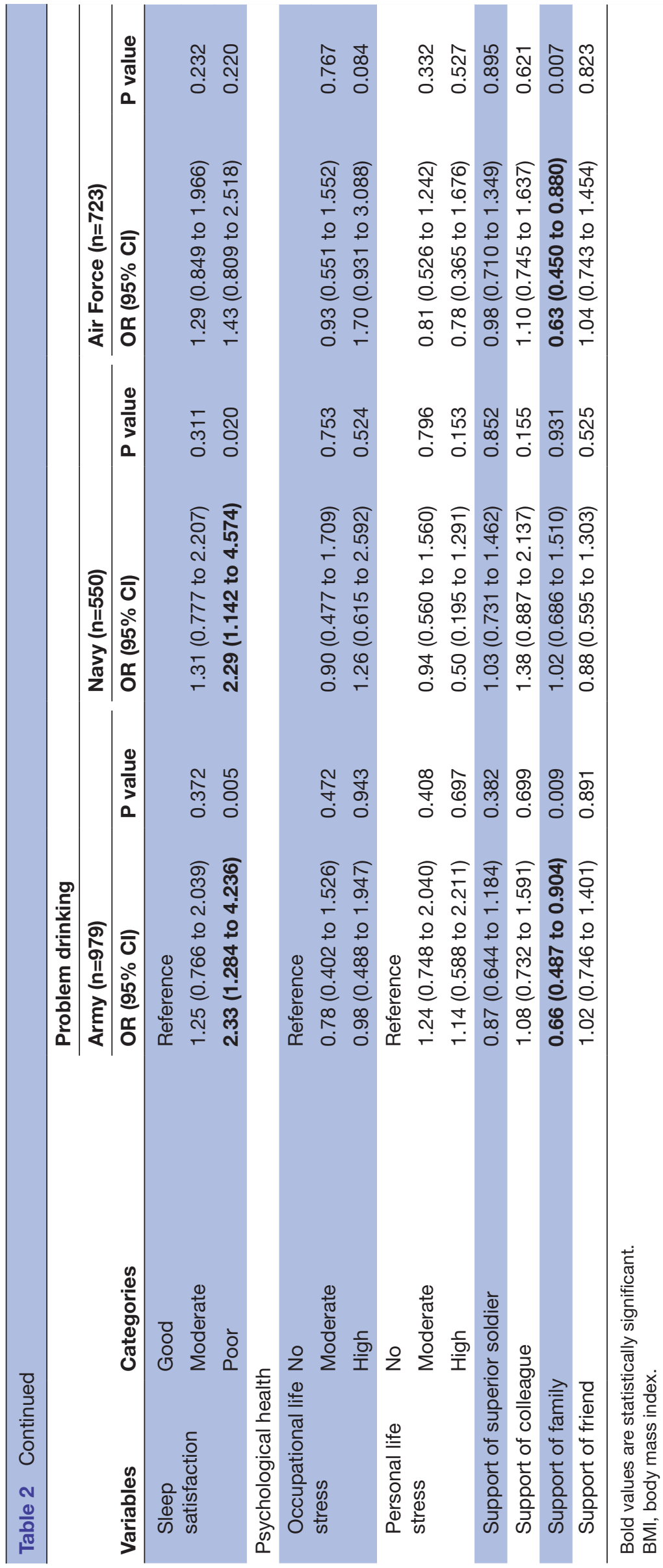


the prevalence of problem alcohol drinking among male professional military personnel was lower compared with previous research but differed by military service type. When the cut-off value was 10 points on the AUDIT-K score, $25.9 \%$ of total participants were problem alcohol drinkers, with $16.4 \%$ in the Army, $34.5 \%$ in the Navy, and $32.1 \%$ in the Air Force in this study. Kim and Jeong found that $32.5 \%$ of military personnel (enlisted soldiers, non-commissioned officers and officers) were problem alcohol drinkers, with an AUDIT cut-off value of 8 points. ${ }^{7}$ The present prevalences of problem alcohol drinking in the Navy and Air force were similar to those reported by Kim and Jeong, ${ }^{7}$ despite this study using a higher cut-off. Differences in prevalence of problem alcohol drinking among military service types may be due to differences in eligibility criteria for recruitment, occupational roles or drinking cultures.

After the Law for the Promotion of Nation's Health was enacted in 1995, the military has been conducting various health promotion projects for smoking cessation, 'stop drinking' and obesity management since $2000 .{ }^{22}$ According to the Military Health Promotion Instruction, the stop drinking project provides educational and promotional content that can be applied to anyone, focusing on health problem that may arise from drinking. ${ }^{31}$ However, the stop drinking project was ineffective, owing to the permissive drinking culture ${ }^{712}$ in which alcohol is recognised as a medium for unity among military members. Furthermore, such culture is a factor that hinders recognition of the severity of problem alcohol drinking. Thus, to successfully conduct projects related to the use of alcohol, nursing officers should regularly check the drinking status of soldiers using AUDIT-K, find people who engage in problem alcohol drinking, and provide them with individual interventions. In addition, as the military is a hierarchy organisation, we should conduct health promotion projects to change the minds of commanders about drinking and the drinking culture that reflect the occupational characteristics and environment of the military service types.

The major finding of the current study was that factors associated with problem alcohol drinking differed among military service types. The associated factors were sleep satisfaction and support from family for the Army, smoking status and sleep satisfaction for the Navy, and marital status, smoking status, subjective oral health status and support from family for the Air Force. Among these factors, health behaviour factors, which can be modifiable, were sleep satisfaction, smoking status and subjective oral health status. First, participants with poor sleep satisfaction had more problem alcohol drinking than those with good sleep satisfaction in the Army and Navy. This result is consistent with previous studies. ${ }^{32} 33$ Alcohol can cause a reduction in sleep-onset latency, waking after sleep onset, rapid eye movement sleep changes ${ }^{32} 33$ and deterioration in sleep quality. ${ }^{33}$

Second, current smokers in the Navy and past and current smokers in the Air Force had higher problem alcohol drinking than non-smokers. This finding is similar to Park's finding that smokers had 4.78 times higher problem alcohol drinking compared with nonsmokers in a study using KNHANES in $2007-2008,{ }^{34}$ and to Chung and Joung's finding that current smokers had 3.10 times higher hazardous alcohol use compared with non-smokers in the 2009 Korean Community Health Survey. ${ }^{35}$ The result was also similar in Kim and Jeong's study, ${ }^{7}$ in which the smoking status of officers and noncommissioned officers was associated with problem alcohol drinking. The result implies that linking alcohol drinking and smoking projects would be more effective than running separate projects.

Third, people with poor subjective oral health were more likely to engage in problem alcohol drinking than those with good subjective oral health in the Air Force. Hyeong and Lee's study using the 2013-2014 KNHANES showed that higher alcohol consumption and frequency of binge drinking was linked to higher periodontitis. ${ }^{36}$ Kim et al also found that periodontal status was significantly associated with harmful alcohol use. ${ }^{37}$ Therefore, dental check-up care needs to be included when nursing officers conduct alcohol-related health interventions for military personnel.

Regarding individual characteristics, in the current study, those who were single were less likely to engage in problem alcohol drinking than those who were married and living with their family in the Air Force. One possible explanation of this finding is that single military personnel in the Air Force engage less in problem alcohol drinking because they have to go through intense flight training and evaluation to become a pilot.

Support from family was identified as an associated psychological health factor in the Army and Air Force. Social support enhances people's belief in their ability to cope effectively and results in fewer negative effects when faced with stress. ${ }^{38}$ Previous studies have shown that family-related stress increases problem alcohol drinking. ${ }^{715}$ Professional military personnel in particular need support from their families as they may lack stability in their lives owing to frequent moving and separation from their families. ${ }^{39}$ Thus, it is necessary to include family support interventions when carrying out alcoholrelated health promotion projects.

Despite its strengths and implications, this study has some limitations. First, it was difficult to identify causes and consequences in the relationship between alcohol drinking and associated factors. Second, since this study was based on secondary data analysis, there was limited information on some factors affecting alcohol drinking such as combat exposure experience. Third, the Navy and Air Force had a large difference in the number of samples by rank. Moreover, the samples were different from the actual proportion of ranks in each military service type. Therefore, the results of this study have limited generalisation. Fourth, this study was conducted through a selfreport survey, which may have resulted in reporting bias, especially for questions on alcohol drinking. Despite these 
limitations, this study is meaningful in suggesting which factors affect problem alcohol drinking among male professional military personnel in South Korea according to military service characteristics, considering differences in work environment and culture. The results reveal differences in factors associated with problem alcohol drinking by military service type. Thus, when implementing projects to address problem alcohol drinking, it is necessary to ensure that service-type-specific factors are considered for integrated management.

\section{CONCLUSION}

The characteristics of each military service should be considered when conducting alcohol-related health interventions because factors affecting problem alcohol drinking for each type of military service were different. In addition, alcohol-related health interventions could be effective if they are conducted with other health interventions for smoking, sleeping and oral health.

Contributors JP, EJ and EB participated in the study design. EJ and EB conducted the data analysis. JP and EJ were the main authors responsible for drafting the manuscript.

Funding This work was supported by the 2018 Faculty-Student Research Fund granted from the Mo-Im Kim Nursing Research Institute, College of Nursing at Yonsei University (6-2017-0203).

Competing interests None declared.

Patient and public involvement Patients and/or the public were not involved in the design, or conduct, or reporting, or dissemination plans of this research.

Patient consent for publication Not required.

Ethics approval The Military Health Survey was approved by the Korea Armed Forced Medical Command's Institutional Review Board (AFMC-15060-IRB-15-049). The raw data of this study excluded personally identifiable information. This study was approved by the Severance Hospital Yonsei University Health System Human Research Protection Centre (Y-2019-0073).

Provenance and peer review Not commissioned; externally peer reviewed.

Data availability statement No data are available. Data sharing is not applicable to this article as the data are managed by the military authority.

Open access This is an open access article distributed in accordance with the Creative Commons Attribution Non Commercial (CC BY-NC 4.0) license, which permits others to distribute, remix, adapt, build upon this work non-commercially, and license their derivative works on different terms, provided the original work is properly cited, appropriate credit is given, any changes made indicated, and the use is non-commercial. See: http://creativecommons.org/licenses/by-nc/4.0/.

ORCID iD

Eunyoung Jung http://orcid.org/0000-0002-0585-4625

\section{REFERENCES}

1 OECD. Health at a glance 2017: OECD indicators. Paris: OECD Publishing, 2017.

2 Korea Center for Disease Control and Prevention. The seventh Korea National health and nutrition examination survey, 2017.

3 WHO. Global status report on alcohol and health, 2018. Available: https://www.who.int/substance_abuse/publications/global_alcohol_ report/en/

4 Joe $\mathrm{KH}$, Chai SH, Park AR, et al. Optimum cut-off score for screening of hazardous drinking using the Korean version of alcohol use disorder identification test (AUDIT-K). J Korean Acad Addict Psych 2009;13:34-40.
5 SW A, Lee JH, Park SS. A study on problem drinking status and measures of criminal activity. Korean Assoc Add Crime Rev 2014;4:80-101.

6 Hyun-young O. A society of binge drinking. Chosun, 2016. Available: https://news.chosun.com/site/data/html_dir/2016/05/13/ 2016051301865.html

$7 \mathrm{Kim} \mathrm{BJ}$, Jeong AS. Associated factors of problem drinking and patterns of drinking among male military personnel in Korea. $J$ Korean Alcohol Sci 2011;12:1-16.

8 Woodruff SI, Hurtado SL, Simon-Arndt CM. U.S. Marines' perceptions of environmental factors associated with alcohol binge drinking. Mil Med 2018;183:e240-5.

9 Barlas FM, Higgins WB, Pflieger JC, et al. 2011 health related behaviors survey of active duty military personnel. Washington: Department of Defense, 2013.

10 Stahre MA, Brewer RD, Fonseca VP, et al. Binge drinking among U.S. active-duty military personnel. Am J Prev Med 2009;36:208-17.

11 American Addiction Center. Problem drinking vs. alcoholism. Available: https://www.alcohol.org/alcoholism/or-is-it-just-a-problem/ [Accessed 30 Apr 2020].

12 Fear NT, Iversen A, Meltzer $\mathrm{H}$, et al. Patterns of drinking in the UK armed forces. Addiction 2007;102:1749-59.

13 Hong J, Kim J, Kim O. Factors influencing problem drinking of male drinkers according to life cycle. Korean J Adult Nurs 2014;26:139-48.

14 Jeon GS, Lee HY. Associated factors of binge drinking and problem drinking among Korean men and women. Korean J Health Educ Prom 2010;27:91-103.

15 Morgan JK, Brown J, Bray RM. Resilience as a moderating factor between stress and alcohol-related consequences in the army national guard. Addict Behav 2018;80:22-7.

16 Cucciare MA, Sadler AG, Mengeling MA, et al. Associations between deployment, military RANK, and binge drinking in active duty and Reserve/National Guard US servicewomen. Drug Alcohol Depend 2015;153:37-42.

17 Waller M, McGuire ACL, Dobson AJ. Alcohol use in the military: associations with health and wellbeing. Subst Abuse Treat Prev Policy 2015;10:27-37.

18 Rona RJ, Fear NT, Hull L, et al. Mental health consequences of overstretch in the UK armed forces: first phase of a cohort study. BMJ 2007;335:603-10.

19 Jang JS, Kim JH, Kim HS, et al. Analysis of factors related to health behavior of the ROKAF pilots. Korean J Aerospace Environ Med 2002;12:19-27.

20 Jo DS. Research study on the factors related to the health of naval officers [master's thesis]. Seoul: Seoul National University, 2018.

$21 \mathrm{Kim}$ S-Y, Ko I-S. Association among health behaviors, occupational stress and obesity of male army officers. KJMNR 2016;34:30-41.

22 Korea Army Headquarters. History of nurse corps branch for 60 years, 2009.

23 Ministry of Government Legislation. Law for the promotion of Nation's health, 2019.

$24 \mathrm{Kim} \mathrm{Y,} \mathrm{Oh} \mathrm{E-geum.} \mathrm{Health} \mathrm{behavior} \mathrm{and} \mathrm{quality} \mathrm{of} \mathrm{life} \mathrm{among} \mathrm{Korean}$ navy. KJMNR 2015;33:53-66.

25 WHO. The alcohol use disorders identification test: guidelines for use in primary health care, 2001.

26 Kim C-G, Kim JS, Jung J-G, et al. Reliability and validity of alcohol use disorder dentification Test-Korean revised version for screening at-risk drinking and alcohol use disorders. Korean J Fam Med 2014;35:2-10.

27 WHO. The Asian-Pacific perspective: redefining obesity and its treatment, 2000

28 Korea Center for Disease Control and Prevention. The sixth Korea National health and nutrition examination survey (KNHANES) 20132015.

29 Lee BO, Lee CH, Lee PG, et al. Development of Korean version of alcohol use disorders identification test (AUDIT-K): its reliability and validity. J Korean Acad Addict Psych 2000;4:83-92.

30 Yoon S-J, Kim H-J, Doo M. Association between perceived stress, alcohol consumption levels and obesity in Koreans. Asia Pac J Clin Nutr 2016;25:316-25.

31 Ministry of National Defence. Military health promotion instruction, 2019.

32 Ebrahim IO, Shapiro CM, Williams AJ, et al. Alcohol and sleep I: effects on normal sleep. Alcohol Clin Exp Res 2013;37:539-49.

33 Choi SJ, Lee SI, Joo EY. Habitual alcohol consumption and metabolic syndrome in patients with sleep disordered breathing. PLoS One 2016;11:e0161276.

34 Park HJ. Prevalence and related risk factors of problem drinking in Korean adult population. $J$ Korea Acad-Ind Cooperat Soc 2018;19:389-97. 
35 Chung SS, Joung KH. Factors associated with the patterns of alcohol use in Korean adults. Korean J Adult Nurs 2012;24:441-53.

36 Hyeong J-H, Lee Y-H. Gender-Specific association between average volume of alcohol consumption, binge drinking, and periodontitis among Korean adults: the Korea National health and nutrition examination survey, 2013 2014. J Dental Hygiene Sci 2016;16:339-48.
$37 \mathrm{Kim} \mathrm{H}-\mathrm{S}$, Son J-H, Yi H-Y, et al. Association between harmful alcohol use and periodontal status according to gender and smoking. BMC Oral Health 2014;14:73-8.

38 Dixit S, Chauhan VS, Azad S. Social support and treatment outcome in alcohol dependence syndrome in armed forces. J Clin Diagn Res 2015;9:VC01-5.

39 Son SH. Factors affecting organizational effectiveness of professional soldiers: Focused on human relations, social support, job stress [dissertation]. Daejeon: Konyang University, 2017. 